Supporting Information

\title{
Unusual Transannular Cyclization Products of Sarcophytoxide, a 14-Membered Marine Cembranoid: Anomalous Stereochemistry of Epoxide-ketone Rearrangement
}

Keiji Nii, ${ }^{\dagger}$ Keiko Tagami, ${ }^{\dagger}{ }^{+}$Keisuke Matsuoka, ${ }^{\dagger}$ Tatsuo Munakata, ${ }^{\ddagger}$ Takashi Ooi, $^{\dagger}$ and Takenori Kusumi ${ }^{\star \dagger}$ 


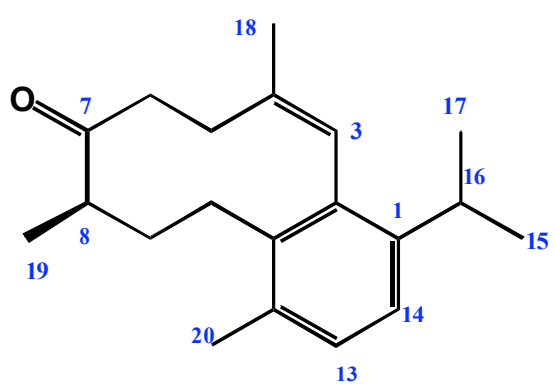

3
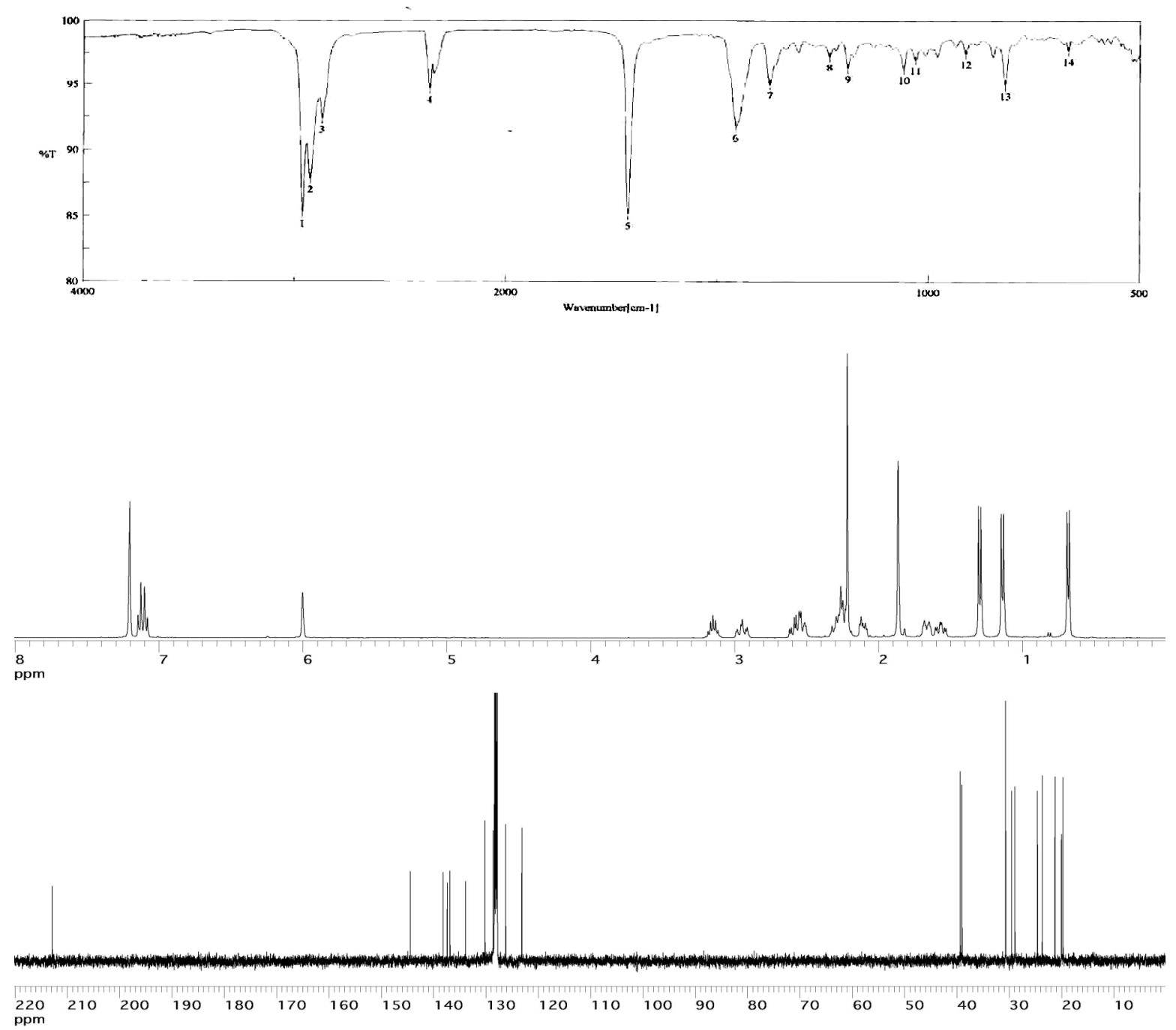

$\operatorname{IR}\left(\mathrm{cm}^{-1}\right) 1710,818 .[\alpha]_{\mathrm{D}}^{27}=+210\left(c 0.17, \mathrm{CHCl}_{3}\right) .{ }^{1} \mathrm{H}-\mathrm{NMR}\left(400 \mathrm{MHz}, \mathrm{C}_{6} \mathrm{D}_{6}\right) \delta: 7.13\left(\mathrm{~d}_{\mathrm{ab}}, 1 \mathrm{H}, J=8.1 \mathrm{~Hz}\right.$, H-14), $7.09\left(\mathrm{~d}_{\mathrm{ab}}, 1 \mathrm{H}, J=8.1 \mathrm{~Hz}, \mathrm{H}-13\right), 6.01(\mathrm{~s}, 1 \mathrm{H}, \mathrm{H}-3), 3.14(\mathrm{sept}, 1 \mathrm{H}, J=6.9 \mathrm{~Hz}, \mathrm{H}-16), 2.95(\mathrm{td}, 1 \mathrm{H}, J=$ 13.4, 3.7 Hz, H-10), 2.57 (td, $1 \mathrm{H}, J=13.4,5.1 \mathrm{~Hz}, \mathrm{H}-5), 2.53(\mathrm{~m}, 1 \mathrm{H}, \mathrm{H}-10$ '), $2.26(\mathrm{~m}, 1 \mathrm{H}, \mathrm{H}-9), 2.25$ (m, $1 \mathrm{H}, \mathrm{H}-8), 2.21(\mathrm{~s}, 3 \mathrm{H}, \mathrm{H}-20), 2.11(\mathrm{~m}, 1 \mathrm{H}, \mathrm{H}-6), 2.10\left(\mathrm{~m}, 1 \mathrm{H}, \mathrm{H}-6^{3}\right), 1.86(\mathrm{~s}, 3 \mathrm{H}, \mathrm{H}-18), 1.66(\mathrm{dt}, 1 \mathrm{H}, J=13.1$, $3.2 \mathrm{~Hz}, \mathrm{H}-5$ '), $1.56(\mathrm{td}, 1 \mathrm{H}, J=12.5,4.6 \mathrm{~Hz}, \mathrm{H}-9$ '), $1.28(\mathrm{~d}, 3 \mathrm{H}, J=6.8 \mathrm{~Hz}, \mathrm{H}-17), 1.13(\mathrm{~d}, 3 \mathrm{H}, J=6.8 \mathrm{~Hz}$, $\mathrm{H}-15), 0.67$ (d, 3H, J = 6.6 Hz, H-19). ${ }^{13} \mathrm{C}-\mathrm{NMR}\left(100 \mathrm{MHz}, \mathrm{C}_{6} \mathrm{D}_{6}\right) \delta 212.8$ (s, C-7), 144.4 (s, C-1), 138.1 (s, C-12), 137.3 (s, C-2), 136.8 (s, C-4), 133.8 (s, C-11), 130.1 (d, C-13), 126.1 (d, C-3), 123.1 (d, C-14), 39.3 (d, C-8), 38.9 (t, C-6), 30.6 (d, C-16), 30.6 (t, C-9), 29.4 (t, C-5), 28.8 (t, C-10), 24.5 (q, C-15), 23.6 (q, C-17), 21.1 (q, C-18), 19.9 (q, C-20), 19.6 (q, C-19). GCMS m/z 284, HREIMS m/z $284.2136(-0.5 \mathrm{mmu})$. 


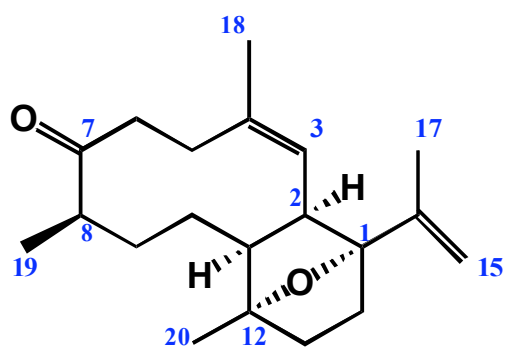

4
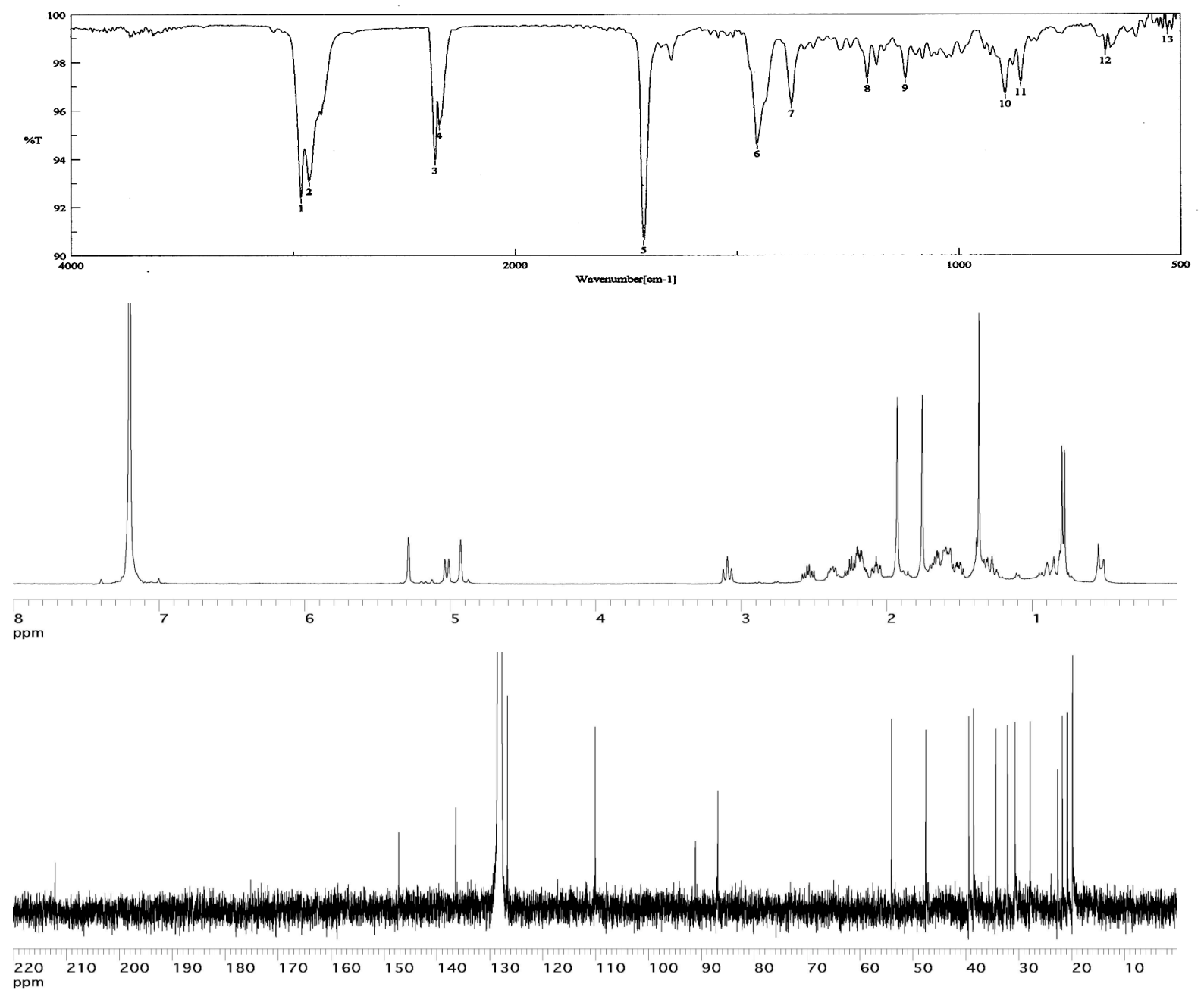

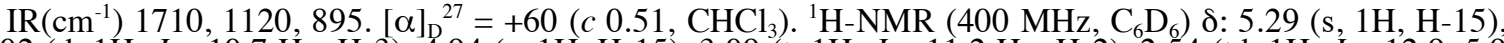
$5.02(\mathrm{~d}, 1 \mathrm{H}, J=10.7 \mathrm{~Hz}, \mathrm{H}-3), 4.94(\mathrm{~s}, 1 \mathrm{H}, \mathrm{H}-15), 3.09(\mathrm{t}, 1 \mathrm{H}, J=11.2 \mathrm{~Hz}, \mathrm{H}-2), 2.54(\mathrm{td}, 1 \mathrm{H}, J=12.9,5.9$ $\mathrm{Hz}, \mathrm{H}-5), 2.36$ (m, 1H, H-8), 2.25 (td, $1 \mathrm{H}, J=12.4,5.8 \mathrm{~Hz}, \mathrm{H}-6), 2.20$ (m, 1H, H-6), 2.18 (m, 1H, H-9), 2.07 (ddd, $1 \mathrm{H}, J=12.0,9.0,3.2 \mathrm{~Hz}, \mathrm{H}-14), 1.92(\mathrm{~s}, 3 \mathrm{H}, \mathrm{H}-17), 1.75(\mathrm{~s}, 3 \mathrm{H}, \mathrm{H}-18), 1.65$ (m, 1H, H-14), 1.58 (m, $1 \mathrm{H}, \mathrm{H}-11), 1.58(\mathrm{~m}, 1 \mathrm{H}, \mathrm{H}-5), 1.50(\mathrm{~m}, 1 \mathrm{H}, \mathrm{H}-13), 1.36(\mathrm{~s}, 3 \mathrm{H}, \mathrm{H}-20), 1.27(\mathrm{~m}, 1 \mathrm{H}, \mathrm{H}-13), 0.88(\mathrm{~m}, 1 \mathrm{H}$, $\mathrm{H}-10), 0.81$ (m, 1H, H-9), 0.78 (d, 3H, $J=7.1 \mathrm{~Hz}, \mathrm{H}-19), 0.35$ (m, 1H, H-10). ${ }^{13} \mathrm{C}-\mathrm{NMR}\left(100 \mathrm{MHz}, \mathrm{C}_{6} \mathrm{D}_{6}\right) \delta$ : 212.0 (s, C-7), 147.8 (s, C-16), 136.3 (s, C-4), 126.4 (d, C-3), 109.9 (t, C-15), 91.0 (s, C-1), 86.8 (s, C-12), 53.9 (d, C-11), 47.5 (d, C-2), 39.3 (d, C-8), 38.5 (t, C-6), 34.3 (t, C-9), 32.0 (t, C-14), 30.6 (t, C-13), 27.8 (t, C-5), 22.6 (q, C-18), 21.7 (t, C-10), 20.8 (q, C-20), 19.7 (q, C-17), 19.7 (q, C-19). GCMS m/z 302, HREIMS $\mathrm{m} / \mathrm{z} 302.2239(-0.7 \mathrm{mmu})$. 


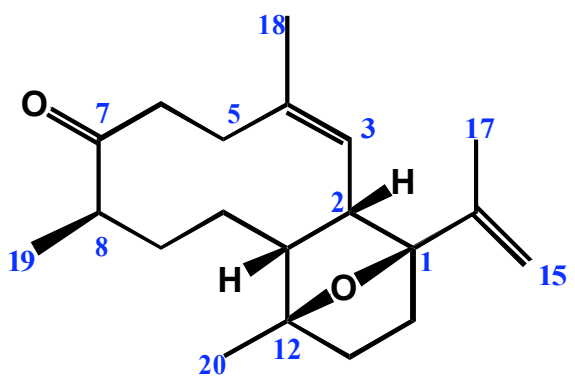

5
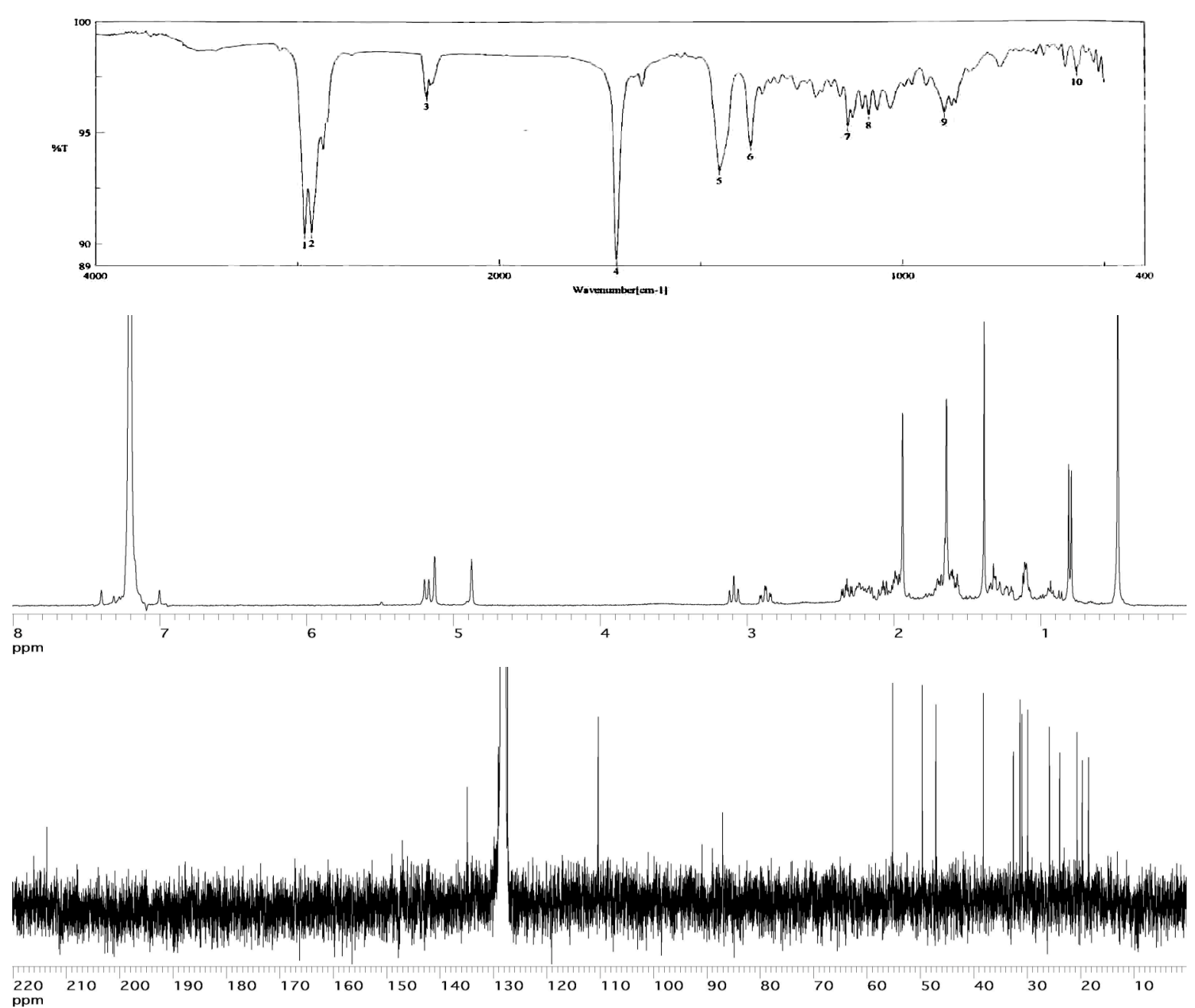

$\operatorname{IR}\left(\mathrm{cm}^{-1}\right) 1710,1135,896 .[\alpha]_{\mathrm{D}}{ }^{24}=+40\left(c 0.38, \mathrm{CHCl}_{3}\right){ }^{1} \mathrm{H}-\mathrm{NMR}\left(400 \mathrm{MHz}, \mathrm{C}_{6} \mathrm{D}_{6}\right) \delta: 5.18(\mathrm{~d}, 1 \mathrm{H}, J=11.7$ $\mathrm{Hz}, \mathrm{H}-3), 5.13(\mathrm{~s}, 1 \mathrm{H}, \mathrm{H}-15), 4.87(\mathrm{~s}, 1 \mathrm{H}, \mathrm{H}-15), 3.09(\mathrm{t}, 1 \mathrm{H}, J=11.7 \mathrm{~Hz}, \mathrm{H}-2), 2.87(\mathrm{td}, 1 \mathrm{H}, J=13.3,3.5 \mathrm{~Hz}$ $\mathrm{H}-5), 2.32$ (ddd, $1 \mathrm{H}, J=13.7,10.7,3.9 \mathrm{~Hz}, \mathrm{H}-6), 2.22(\mathrm{~m}, 1 \mathrm{H}, \mathrm{H}-11), 2.17$ (m, 1H, H-8), 2.06 (m, 1H, H-14), $2.00(\mathrm{~m}, 1 \mathrm{H}, \mathrm{H}-9), 1.97$ (m, 1H, H-6), $1.92(\mathrm{~s}, 3 \mathrm{H}, \mathrm{H}-17), 1.70(\mathrm{~m}, 1 \mathrm{H}, \mathrm{H}-14), 1.63(\mathrm{~s}, 3 \mathrm{H}, \mathrm{H}-18), 1.62(\mathrm{~m}, 1 \mathrm{H}$, $\mathrm{H}-13), 1.57$ (m, 1H, H-5), 1.37 (s, 3H, H-20), 1.31 (m, 1H, H-13), 1.21 (m, 1H, H-9), $1.11(\mathrm{~m}, 2 \mathrm{H}, \mathrm{H}-10)$, 0.79 (d, 3H, $J=7.1 \mathrm{~Hz}, \mathrm{H}-19) .{ }^{13} \mathrm{C}-\mathrm{NMR}\left(100 \mathrm{MHz}, \mathrm{C}_{6} \mathrm{D}_{6}\right) \mathrm{\delta}: 213.5$ (s, C-7), 147.7 (s, C-16), 134.8 (s, C-4), 127.2 (d, C-3), 110.3 (t, C-15), 90.9 (s, C-1), 87.0 (s, C-12), 55.1 (d, C-11), 49.6 (d, C-8), 47.0 (d, C-2), 38.1 (t, C-6), 32.5 (t, C-9), 31.3 (t, C-14), 30.9 (t, C-13), 29.8 (t, C-5), 25.8 (t, C-10), 23.8 (q, C-18), 20.6 (q, C-20), 19.2 (q, C-17), 18.4 (q, C-19). GCMS m/z 302, HREIMS m/z 302.2239 (-0.7 mmu). 


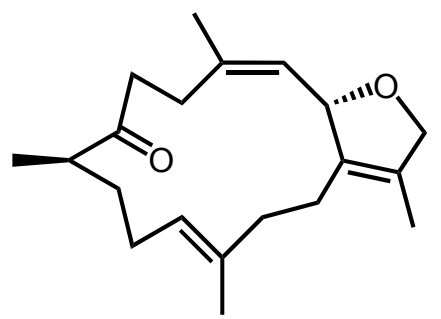

6
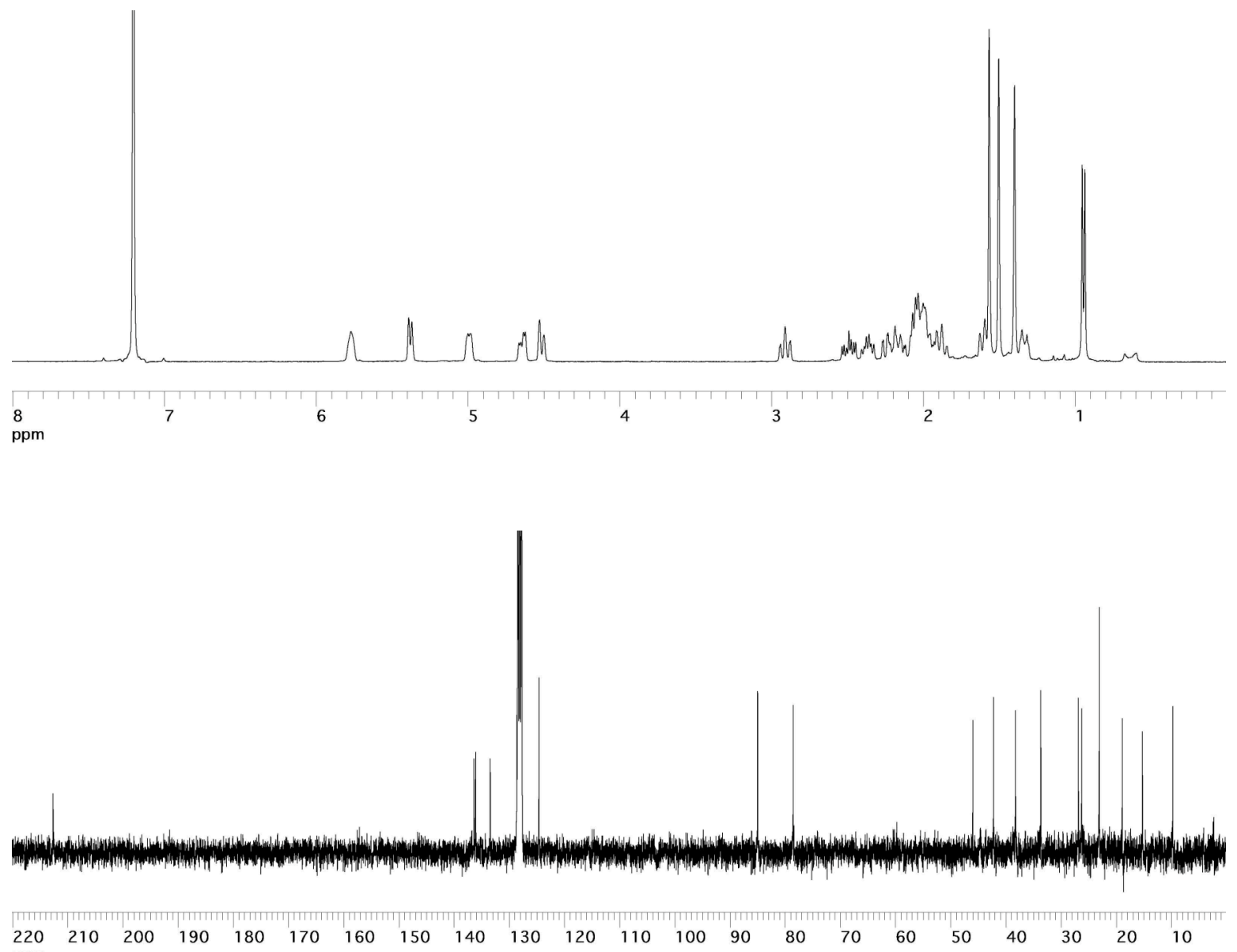

ppm

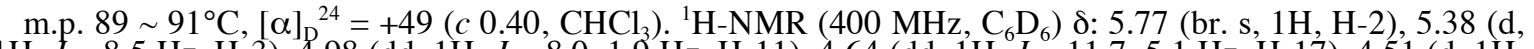
$1 \mathrm{H}, J=8.5 \mathrm{~Hz}, \mathrm{H}-3), 4.98(\mathrm{dd}, 1 \mathrm{H}, J=8.0,1.9 \mathrm{~Hz}, \mathrm{H}-11), 4.64(\mathrm{dd}, 1 \mathrm{H}, J=11.7,5.1 \mathrm{~Hz}, \mathrm{H}-17), 4.51(\mathrm{~d}, 1 \mathrm{H}$, $J=11.7 \mathrm{~Hz}, \mathrm{H}-17), 2.90(\mathrm{td}, 1 \mathrm{H}, J=13.2,2.4 \mathrm{~Hz}, \mathrm{H}-5), 2.48(\mathrm{ddd}, 1 \mathrm{H}, J=18.5,12.2,5.6 \mathrm{~Hz}, \mathrm{H}-6), 2.36$ (m, 1H, H-14), 2.24 (td, $1 \mathrm{H}, J=18.5,12.7,2.9 \mathrm{~Hz}, \mathrm{H}-6$ ), 2.15 (overlap, 1H, H-9), 2.06 (overlap, 1H, H-8), 2.02 (overlap, 1H, H-5), 2.01 (overlap, 1H, H-13), 1.97 (m, 2H, H-10), 1.86 (m, 1 H, H-13), 1.59 (m, 1H, H-14), 1.56 (s, 3H, H-18), 1.49 (s, 3H, H-20), 1.39 (s, 3H, H-15), 1.33 (m, 1H, H-9), 0.93 (d, 3H, J = 6.8 Hz, H-19). ${ }^{13} \mathrm{C}-\mathrm{NMR}\left(100 \mathrm{MHz}, \mathrm{C}_{6} \mathrm{D}_{6}\right)$ 8: 212.6 (s, C-7), 136.3 (s, C-12), 136.0 (s, C-4), 133.4 (s, C-1), 128.5 (s, C-16), 128.3 (d, C-3), 124.5 (d, C-11), 84.9 (d, C-2), 78.4 (t, C-17), 45.9 (d, C-8), 42.1 (t, C-6), 38.2 (t, C-13), 33.6 (t, C-9), 26.8 (t, C-10), 26.2 (t, C-5), 23.0 (q, C-18), 23.0 (t, C-14), 18.8 (q, C-19), 15.2 (q, C-20), 9.2 (q, C-15). EIMS $m / z$ 302, HREIMS $m / z 302.2247$ (+0.2 mmu). 

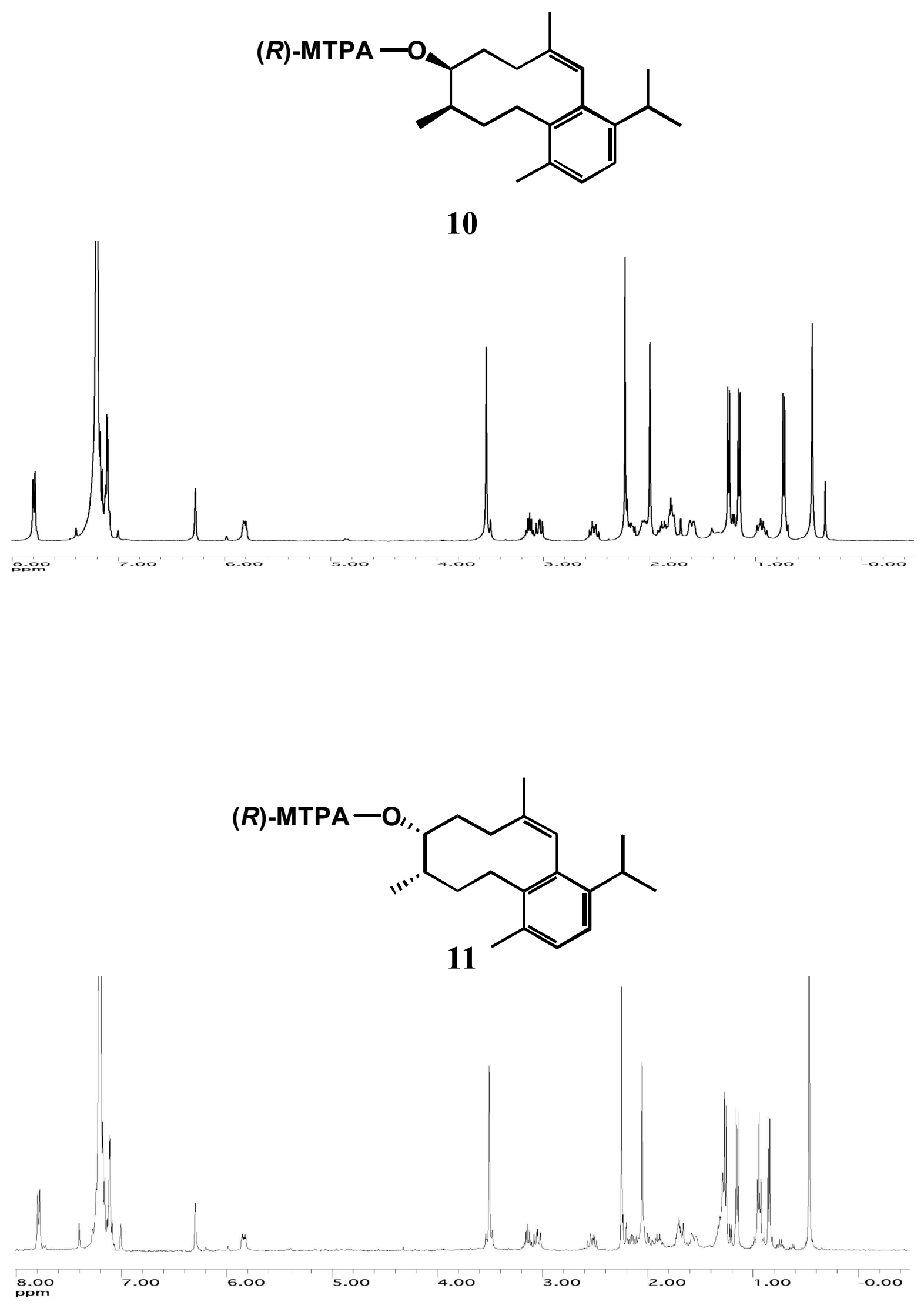

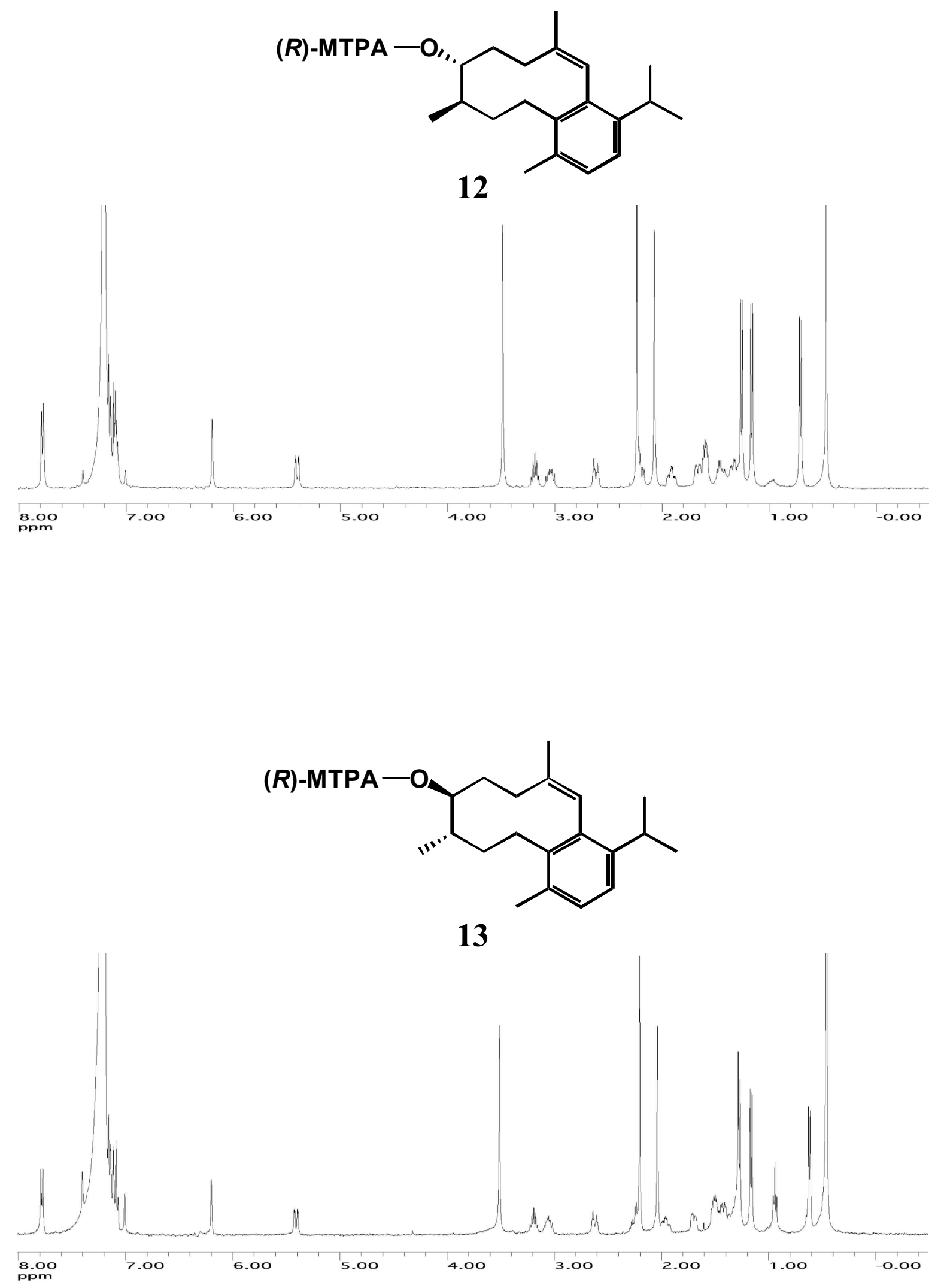


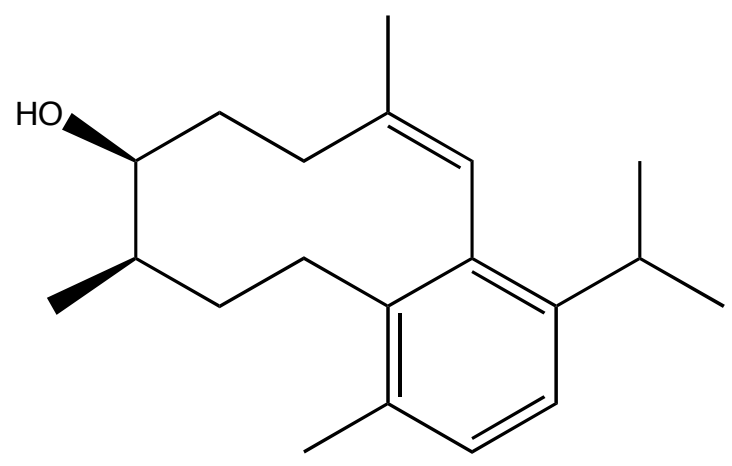

10

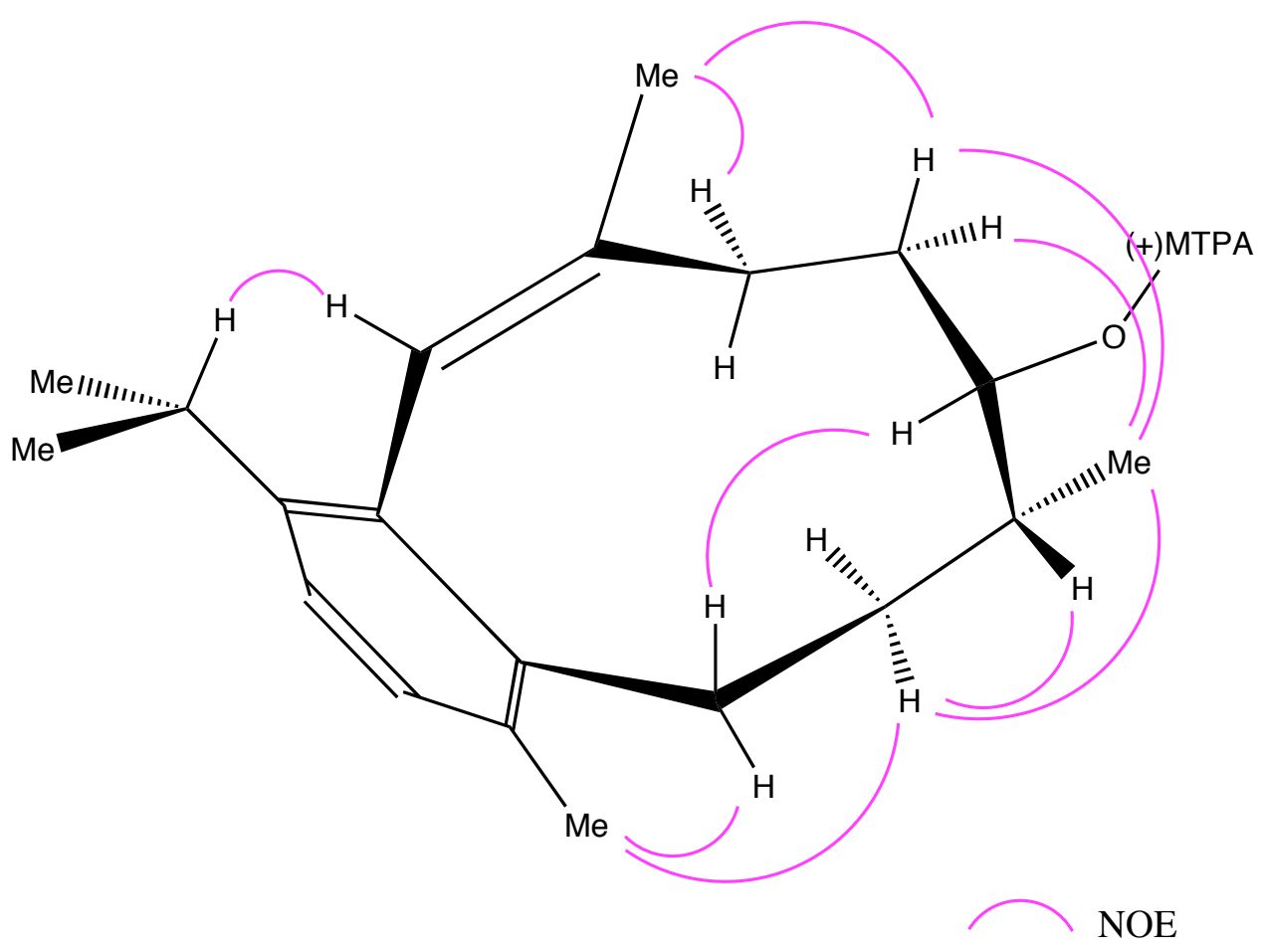

NOESY data obtained for $\mathbf{1 0}$ 


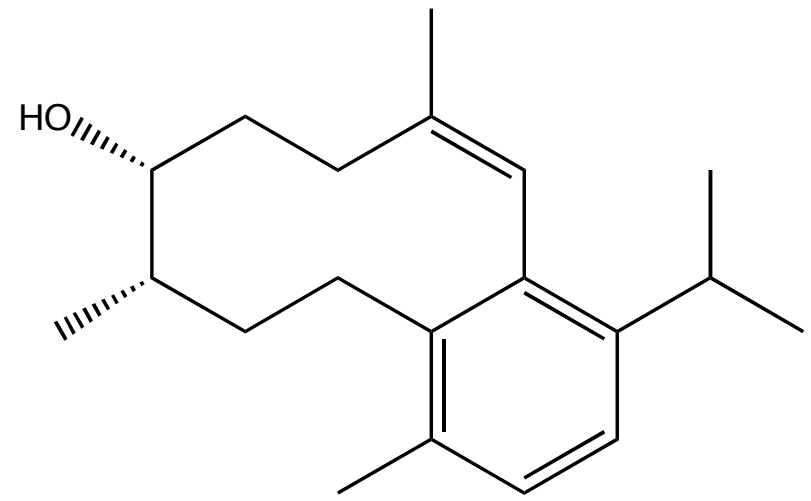

11

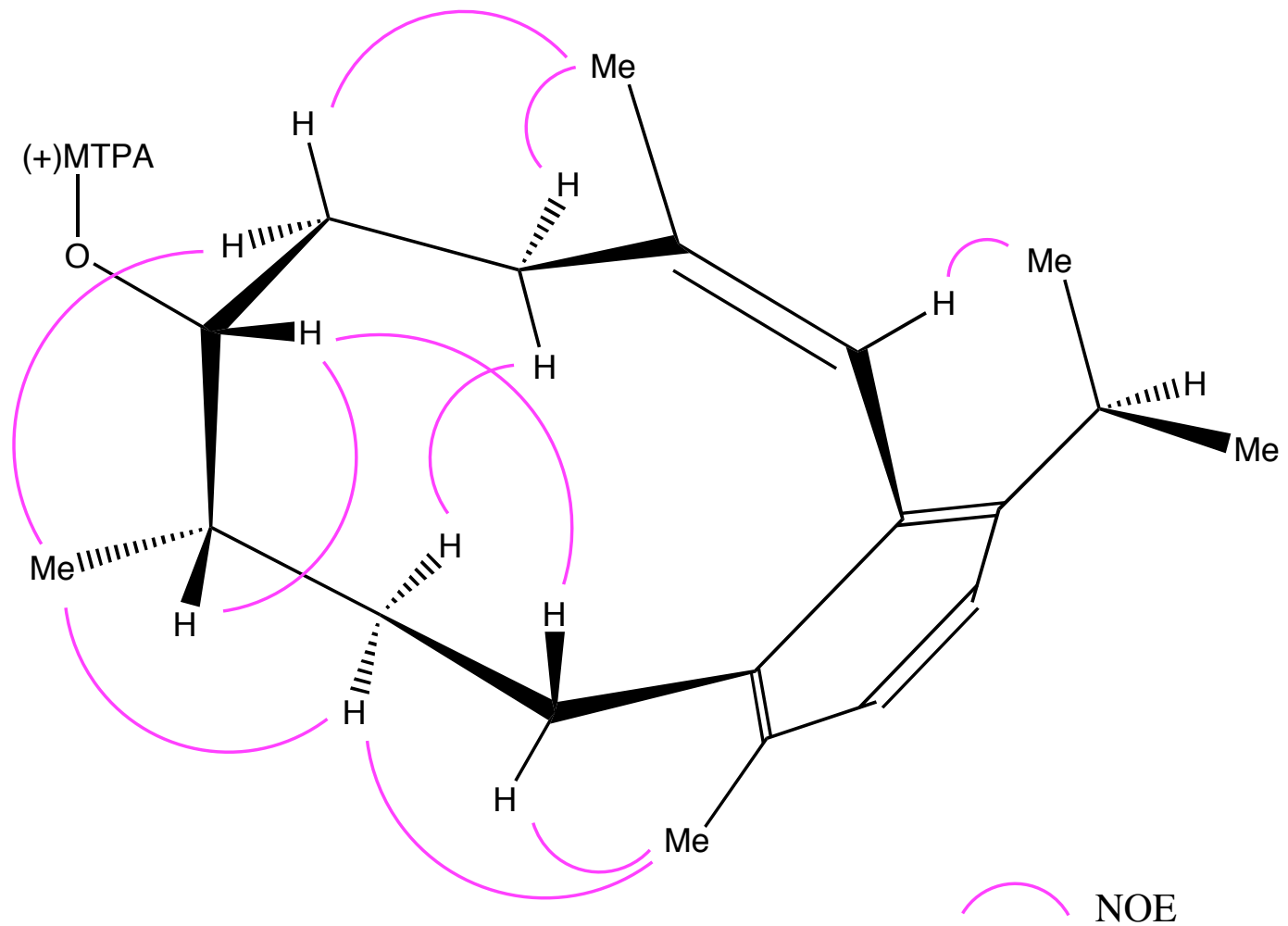

NOESY data obtained for $\mathbf{1 1}$ 


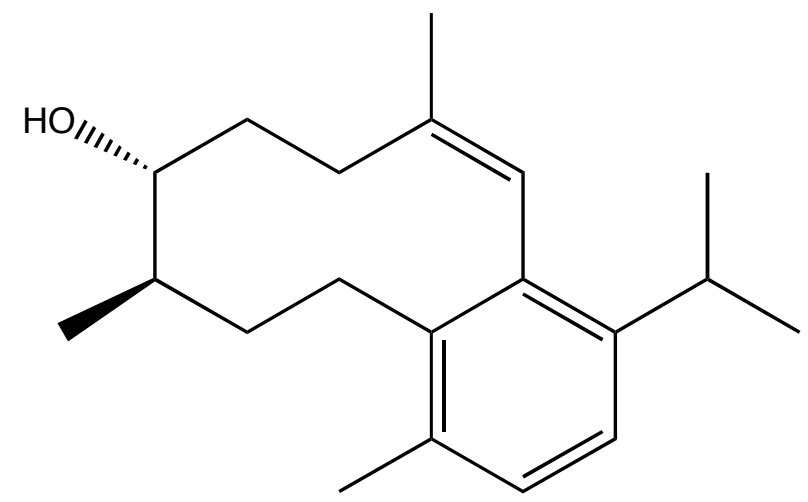

12

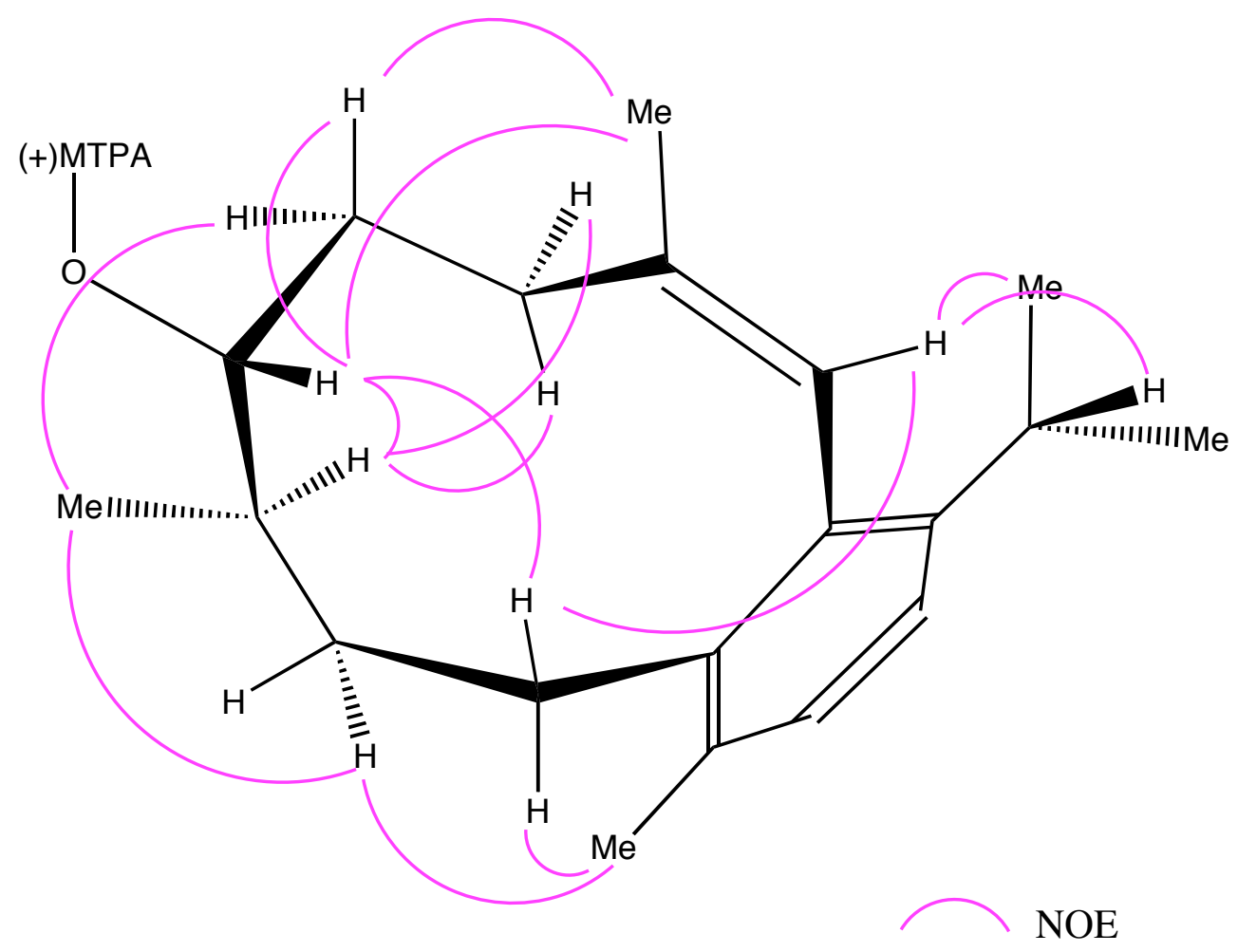

NOESY data obtained for $\mathbf{1 2}$ 


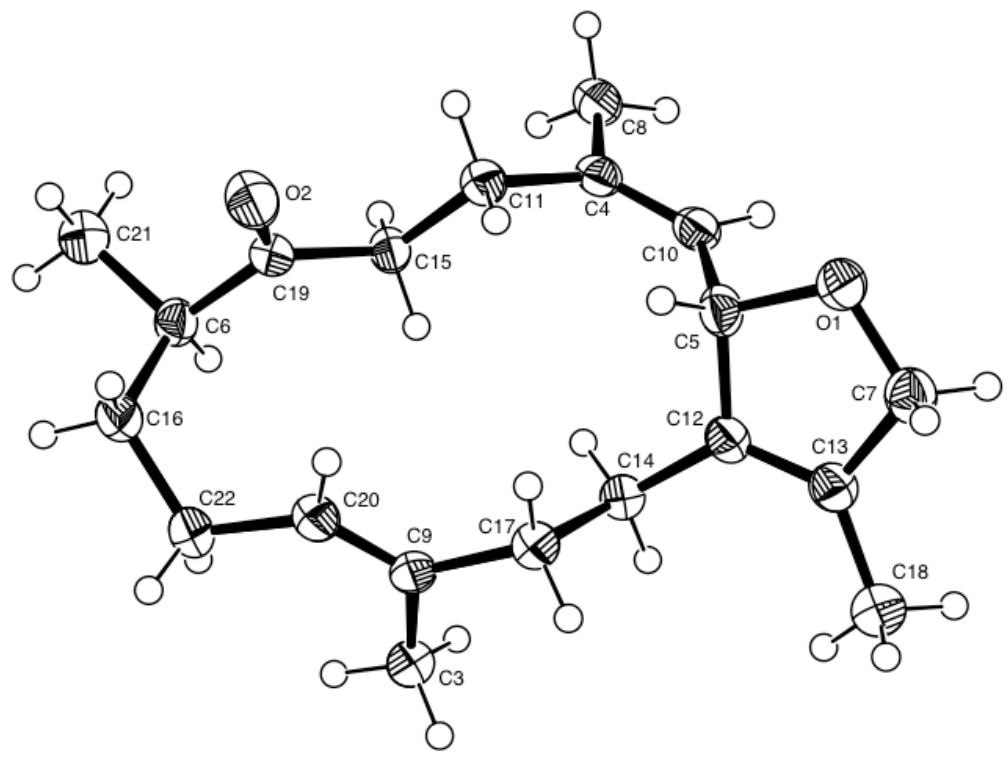

X-ray structure of compound $\mathbf{6}$

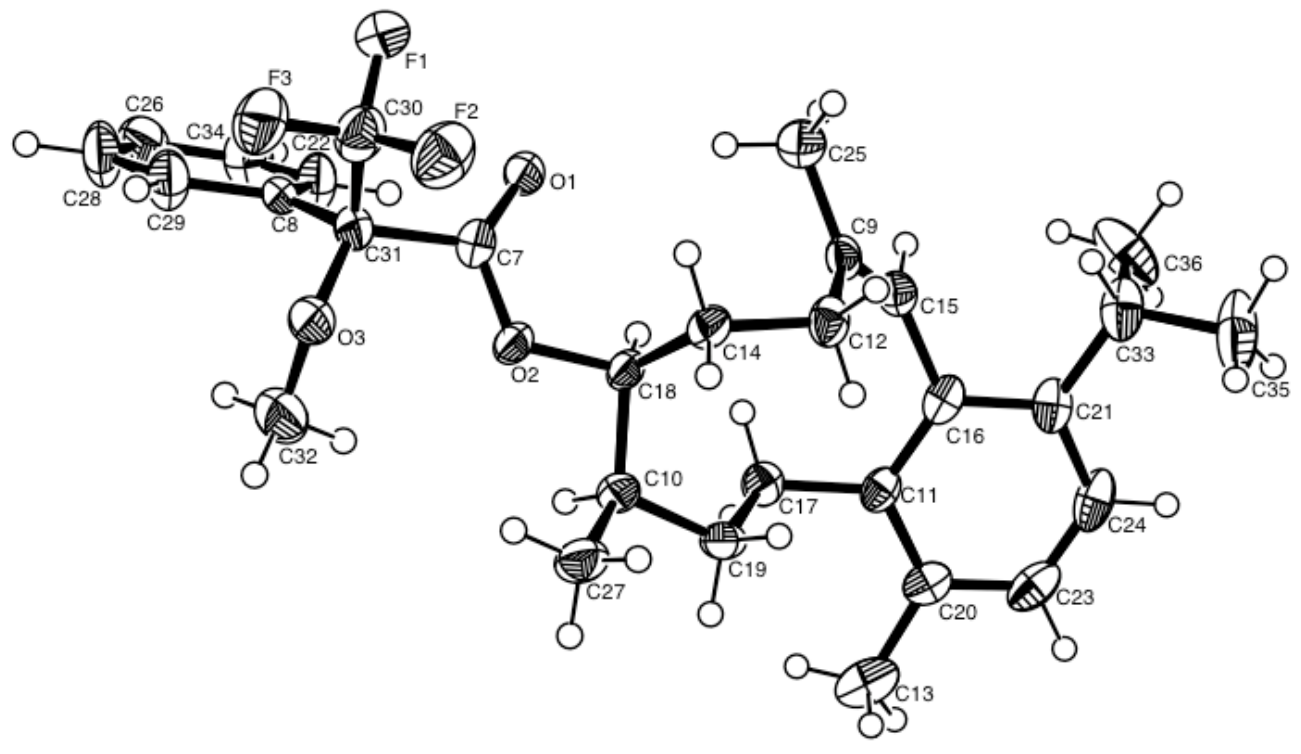

X-ray structure of compound $\mathbf{1 0}$ 